phosphate buffer ( $\mathrm{pH} 7 \cdot 2$ ). The mobile phase $\mathrm{B}$ consisted of $22 \cdot 2 \%$ acetonitrile in methanol. The increments of buffer $B$ in the gradient programme were $6.5 \%, 12 \cdot 8 \%$, $15 \cdot 5 \%, 27 \cdot 0 \%, 39 \cdot 0 \%, 41 \cdot 0 \%$, and $59.5 \%$ at $5 \cdot 5,6 \cdot 0,13 \cdot 0,13 \cdot 5,14 \cdot 0,19 \cdot 5$, and $22 \cdot 5$ minutes, respectively. The analysis was carried out at a flow rate of $1.5 \mathrm{ml} / \mathrm{min}$.

Before analysis 20 and $5 \mu \mathrm{l} \mathrm{CSF} \mathrm{samples}$ were diluted with an internal standard of 20 and $95 \mu \mathrm{l} a$-aminoadipic acid $(5 \mu \mathrm{M})$ respectively. The reagent mixture was prepared by dissolving $10 \mathrm{mg} \sigma$-phthalaldehyde in $250 \mu \mathrm{l}$ methanol and adding $50 \mu \mathrm{l} 2$-mercaptoethanol in $4.7 \mathrm{ml} 1 \mathrm{M}$ borate buffer (pH 10.5). Derivatives were made by mixing $63 \mu \mathrm{l} o$-phthalaldehyde with a $7 \mu \mathrm{l} \mathrm{sam-}$ ple two minutes before injecting on to the column.

Statistical analysis was by unpaired $t$ test and Mann Whitney $U$ test. Data are given as means (SEM).

Although there were slight decreases in the concentrations of amino acids in patients with essential tremor there were no significant differences in most amino acids (table). The control values were similar to those found by others. ${ }^{4}$

Despite the great number of experimental studies dealing with the aetiology of tremor induced by harmalin, no human data exist yet that directly explain the generation of essential tremor.

The most surprising change in the concentrations of amino acids was that the concentration of serine and glycine was reduced. Glycine can be formed from serine by a reversible folate dependent reaction catalysed by the enzyme, serine trans-hydroxymethylase. Therefore it is concluded that the reduction in the concentration of glycine is a consequence of the decreased concentration of its major precursor, serine. The important role of glycine in decreased inhibition in the CNS has been demonstrated in the mutant spastic mouse. The less severe phenotypes of these autosomal recessive inherited mice are characterised only by tremor of trunks and limbs, raising an interesting comparison with the similar features of essential tremor. ${ }^{5}$

Whereas the concentration of glutamate was not significantly changed, that of aspartate was significantly decreased $(p<0.01)$. The climbing fibres of the cerebellum utilise excitatory amino acids as neurotransmitters ${ }^{67}$ and an increase in the concentration of excitatory amino acids might have been expected if an overactivity of the inferior olive were involved in essential tremor. From experimental studies, however, the expected candidate for this neurotransmitter is glutamate. ${ }^{67}$

This is the first clinical study that draws attention to the possible role of glycine and it is concluded that the decreased concentration of glycine may be involved in essential tremor. Our findings also strengthen the hypothesis that this disease is of central origin.

We thank Dr Miklós Vajda for his gift of automatic injector programme.

JUDIT MÁLLY Department of Neurology, County Fehér, Székesfehérvár, fózsef 8,

H-8001, Hungary MÁRIA BARANYI
Experimental Institute of Hungarian Academy of Science, Budapest Szigony 43,
-1083 , Hungary
Correspondence to: Dr Judit Málly.

1 Rajput AH, Offord KP, Beard CM, Kurland LT. Essential tremor in Rochester, Minnesota: a 45-year study. $f$ Neurol Neurosurg Psychiatry 1984;47:466-70.

2 Rajput AH, Rozdilsky B, Ang L, Rajput A. Clinicopathologic observations in essential tremor: reports six cases. Neurology 1991;41: tremor:

3 Málly J, Stone TW. The effect of theophylline on essential tremor: the role of GABA. Pharmacol Biochem Behav 1991;39:345-51.

4 Gjessing LR, Gjesdahl P, Sjaastad O. The free amino acids in human cerebrospinal fluid. $\mathcal{F}$ Neurochem 1972;19:1807-8.

5 Becker CM. Disorders of the inhibitory glycine receptor: the spastic mouse. FASEB Ұ 1990;4:2767-74.

6 Stone TW. Glutamate as the neurotransmitter of cerebellar cells in the rat: electrophysiological evidence. Br $\Im$ Pharmacol 1979;66: 291-6.

7 Barry J, Gombos G, Vizi ES. Release of $\left({ }^{3} \mathrm{H}\right)$ L-Glutamate and $\left({ }^{3} \mathrm{H}\right) \mathrm{L}-$ Glutamine in rat cerebellum slices: a comparison of the effect of veratridine and electrical stimulaeffect of veratridine and electrical

\section{Persistent hemiballism in Parkinson's} disease

Ballism has been regarded traditionally as a unique hyperkinetic movement disorder with clear anatomical localisation. In humans and other primates, lesions of the subthalamic nucleus cause contralateral hemiballism. This rather neglected small anatomical structure has lately attracted interest in connection with Parkinson's disease, as it has been shown in primates exposed to 1-methyl-4-phenyl-1,2,3,6tetrahydropyridine (MPTP), that the indirect motor loop from the striatum via the lateral segment of the globus pallidus and the subthalamic nucleus may be overactive, whereas the converse is true for the GABAergic projection from the globus pallidus to the thalamus.

It is on this basis that experimental anatomical lesions of the subthalamic nucleus or neutralisation of its activity by high frequency electrical stimulation or by pharmacological agents such as $\mathrm{N}$-methylD-aspartate (NMDA) antagonists, have been tried in Parkinson's disease or the MPTP models. Such lesions can reverse parkinsonian symptoms in the contralateral limbs.

In patients with Parkinson's disease, lesions in the subthalamic nucleus can reverse parkinsonism contralaterally. ${ }^{2} \mathrm{We}$ recently treated a patient with Parkinson's disease in whom a lesion in the subthalamic nucleus was expressed by sustained violent hemiballism. The patient was a 60 year old right handed man. His history showed arterial hypertension and non-insulin dependent diabetes mellitus, both controlled pharmacologically. $\mathrm{He}$ had gait disturbances and recurrent falls. When examined by a neurologist, hypomimia, symmetric limb rigidity that increased on contralateral activation, and a short step gait were noted. He had no tremor and showed a mild central left facial weakness accompanied by mild pyramidal signs. Treatment with levodopa and carbidopa and selegiline was beneficial.

About one year later, sudden involuntary violent ballistic movements of the left limbs appeared. On examination, there was rigid limb tone on the right whereas on the left the tone was normal. Tendon reflexes were brisker on the left and the plantar response was extensor. CT showed a haemorrhage in

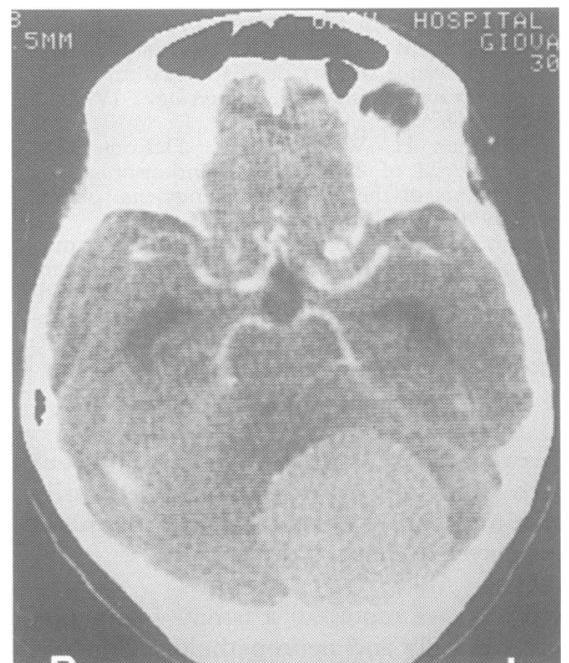

Figure Unenhanced CT performed about six hours after the appearance of left hemiballism. Note the haemorrhage in the right subthalamus.

the right subthalamic nucleus (figure).

The ballistic movements continued despite withdrawal of antiparkinsonian drugs. Increasing doses of haloperidol up to $30 \mathrm{mg} /$ day were not beneficial but aggravated the parkinsonian symptoms, mainly bradykinesia. Clonazepam was added at a dose of $1 \mathrm{mg}$ thrice daily, which minimally reduced the hemiballism but had to be withdrawn because of intolerable somnolence.

Three months after the event, the patient still had hemiballistic movements, although somewhat attenuated. At this time the muscle tone in the left limbs was still normal, by contrast with the rigidity on the right.

The co-occurrence of Parkinson's disease and a lesion in the subthalamic nucleus in the same patient is a coincidental event of low probability, and confirms that destruction of the subthalamic nucleus in a patient with Parkinson's disease can reverse parkinsonian symptoms in the contralateral limbs as suggested by studies in primates. ${ }^{34}$

The persistence of hemiballism for several weeks is unusual. If hemiballism occurs after iatrogenic subthalamic nucleus destruction in monkeys treated with MPTP, it is usually of short duration. Aziz et al, however, in a study of the alleviating effect of lesions of the subthalamic nucleus in parkinsonian monkeys, mentioned one animal in whom hemiballism persisted for at least 16 weeks. ${ }^{4}$ This finding, together with ours, implies that the occurrence, severity, and persistence of hemiballism should be carefully studied before the use of subthalamotomy by iatrogenic anatomical destruction becomes a therapeutic option in Parkinson's disease.

We thank Hagai Bergman MD, PhD, Department of Physiology, Hadassah Medical School, Hebrew University of Jerusalem, for his interest and comments. AMOS D KORCZYN Sackler Faculty of Medicine, Tel-Aviv University, Ramat-Aviv, Israel
Raviv University,

1 Mitchell IJ, Clarke CE, Boyce S, et al. Neural mechanisms underlying parkinsonian symptoms based upon uptake of 2-deoxyglucose in monkeys exposed to 1-methyl-4-phenyl1,2,3,6-tetrahydropyridine. Neuroscience 1989; 32:213-26. 
2 Sellal F, Hirsch E, Lisovoski F, Mutschler V, Collard M, Marescaux C. Contralateral disappearance of parkinsonian signs after subthalamic hematoma. Neurology 1992;42. 255-6.

3 Bergman $H$, Wichmann $T$, DeLong MR Reversal of experimental parkinsonism by lesions of the subthalamic nucleus. Science 1990;249:1436-8.

4 Aziz TZ, Peggs D, Sambrook MA, Crossman AR. Lesion of the subthalamic nucleus for the alleviation of 1-methyl-4-phenyl-1,2,3,6the alleviation of 1-methyl-4-phenyl-1,2,3,6tetrahydropyridine (MPTP) induced parkin-
sonism in the primate. Mov Disord 1991; 6:288-92.

Brain glucose utilisation in a patient with "athymhormia" from a family with autosomal dominant parkinsonism and psychic disturbances

Recently, we reported ${ }^{1}$ a family with a picture clinically and neuropathologically consistent with the syndrome of autosomal dominant parkinsonism associated with psychic disorders (ADPPD). ${ }^{2}$

The psychic disturbances reported in patients with ADPPD have remained ill defined to date, but have often been referred to as "mental depression" or "apathy". In one patient from our family with ADPPD, we were able to further qualify these psychic features, and to assess the underlying brain functional alterations with PET.

In 1987 a 56 year old female patient showed progressive psychic retardation. In 1989 she showed global hypokinesia with slight extrapyramidal hypertonia but no tremor. At that time, a course of levodopa treatment was begun but had soon to be stopped due to total digestive intolerance. In 1990, akinesia was pronounced but extrapyramidal hypertonia was still moderate. Meanwhile, the patient had become more and more indifferent to her surroundings, neglecting everyday chores and dependent for everyday matters on those attending her. She had no complaints except that of being mentally void. She exhibited a flat affect but no anxiety, and did not express any depressive thoughts. She was, however, reluctant to submit to medical examination. In addition to akinesia, examination highlighted an inexpressive facies and moderate extrapyramidal hypertonia of all four limbs without axial hypertonia; examination at a date close to PET showed resting tremor of the lower limbs. There was no oculomotor abnormality.

Two years after onset the neuropsychological testing (limited by the patient's lack of cooperation) showed no significant global intellectual deterioration (Benton's visual retention test, Signoret's cognitive assessment set $=76$, mini mental state $=28$ ), although her memory quotient on the Wechsler memory scale was slightly low at 86 . Verbal fluency was reduced at six words a minute and the verbal span forward slightly reduced at five. No sign of frontal lobe dysfunction could be elicited on Luria's graphic series. There was no grasping. Brain CT, EEG, and copper metabolism were all normal. Questionable global frontal atrophy could be seen on T1 and T2 weighted MRI performed in 1990.

Her two brothers and her only sister had a similar clinical presentation. ${ }^{1}$ Postmortem examination on the two brothers (carried out six and 18 years after the onset of the illness) had shown essentially identical lesions: clearcut cortical atrophy limited to the frontal lobes, massive neuronal and pigment loss in the substantia nigra and other brainstem pigmented nuclei, and moderate neuronal loss in the striatum, pallidum, thalamic nuclei, septal nuclei, and diagonal band of Broca. No Lewy body was found by optical microscopy or immunocytochemical staining with ubiquitine antibodies either in the substantia nigra or in the cortex. Immunocytochemical staining by A4 protein and TAU protein antibodies were negative. No senile plaques, neurofibrillary degeneration, amyloid angiopathy, Pick's bodies, or iron deposition were found. cose (CMRglc) was measured at rest with eyes closed, by means of 18-fluorodeoxyglucose, with a seven slice PET device of high resolution $(5.5 \times 5.5 \times 9 \mathrm{~mm}, \mathrm{x}, \mathrm{y} \mathrm{z}$, model TTV 03, LETI, Grenoble, France) in stereotaxic positioning conditions relative to the Glabella-Inion line. Attenuation correction was carried out by means of a transmission scan. The regions of interest were positioned directly over the MRI cross secmeans of a software enabling MRI data acquired in 3D (FLASH procedure) to be resliced to the PET coordinates with respect to the Glabella-Inion landmarks. Anatomical structures were identified on the MRI relative to the bicommissural line, and with Talairach's stereotaxic atlas of the human brain. The circular (14 mm diameter) regions of interest, 116 in number, aimed at analysing the main functional neocortical and subcortical structures, with averaging over both sides.

The absolute CMRglc values and the relative metabolic indices (region/neocortex) were compared with confidence intervals calculated in a sample of 10 healthy volunteers of mean age 51 (SD 9) years. The confidence intervals for single subject predictions were calculated as mean (SDt), adjusting $t$ to the sample size and the two tailed probability value chosen $(t=2 \cdot 262$ for $9 \mathrm{df}$, and $\mathrm{p}<0.05$ here)

Analysis of the absolute CMRglc values showed no significant abnormality, although the values for all the structures were close to the lower $95 \%$ confidence limit as calculated in our control group. Analysis of the "Region/Neocortex" indices (table) showed diminished values for the lateral prefrontal cortex, which just reached the $p=0.05$ level. The temporal $/$ neocortex index was significantly reduced $(p<0.05)$, and the occipital/neocortex index was significantly raised $(p<0.01)$; other regional
In 1990, the cerebral utilisation of glutions corresponding to the PET planes, by

metabolic indices showed no significant change. A lateral-prefrontal/occipital ratio, calculated to assess the anteroposterior metabolic gradient, was significantly $(p<$ $0.05)$ reduced $(0.78$ for a $95 \%$ lower confidence limit of $0 \cdot 80$ )

Our patient's abnormal behaviour comes nearest to what has been described since the $1920 \mathrm{~s}$ in the French literature as "athymhormia" (in Greek, lack of $\theta v \mu o$ s, affect and $\delta \rho \mu \eta^{\prime}$, drive), initially in psychotic patients and more recently in patients with bilateral lesions of the striatum. ${ }^{3}$ Laplane et $a l^{4}$ described a similar presentation in patients with bilateral lesions of the pallidum or frontal lobe white matter that they called "loss of psychic self-activation" or "psychic akinesia", also described recently after paramedian bilateral thalamic infarct. ${ }^{5}$ Both "athymhormia" and "loss of psychic self-activation" share certain features that suggest frontal lobe dysfunction (for example, mental retardation, reduced initiative), but their hallmark is loss of drive (lack of interest in things of life) and a flat affect (lack of emotional reactivity) without global mental deterioration. Reversal of this behaviour by external stimulation, however, seems less efficient in "athymhormia", 4 which was the case in our patient.

The cerebral metabolic values in our patient showed minor changes, with a marginally significant relative lateral-prefrontal, and significant lateral-temporal hypometabolism, as well as a significant increase in relative metabolism of the occipital cortex, but without metabolic alterations of the precentral cortex or parietal lobe. This pattern suggests an abnormal anteroposterior metabolic gradient, also shown by a significantly reduced lateral-prefrontal/occipital ratio. Because this is the first report of metabolic changes in the brain in ADPPD, our findings can only be compared with previous literature in other related diseases.

Previous studies in patients with psychic akinesia from mainly bipallidal ${ }^{6}$ or bithala$\mathrm{mic}^{5}$ lesions have also reported mild lateral prefrontal hypometabolism, suggesting disruption in the prefronto-striato-pallidothalamo-prefrontal circuit ${ }^{7}$ at different levels results in a similar clinicometabolic picture. Neuropathological data in our patient's two brothers (obtained at an advanced stage) indicate the presence of lesions at several locations in this circuit, with a possible cumulative effect on cortical function; moreover, this loop was presumably deprived of its modulating dopaminergic afferents, as is also the case in progressive

Regional metabolic ratios (region/neocortex)

\begin{tabular}{|c|c|c|}
\hline Region & Patient & Controls (mean ( $95 \%$ confidence intervals)) \\
\hline Frontobasal cortex & 0.91 & $0.94(0.73-1.15)$ \\
\hline Prefrontal lateral cortex & $0.92^{\star}$ & $1.05(0.92-1.17)$ \\
\hline Whole prefrontal cortex & 0.92 & $1.01(0.86-1 \cdot 15)$ \\
\hline Precentral cortex & 1.03 & $1.06(0.89-1.23)$ \\
\hline Whole frontal cortex $\ddagger$ & 0.94 & $1.02(0.88-1.14)$ \\
\hline Whole temporal cortex & $0 \cdot 88^{\star \star}$ & $0.96(0.89-1.01)$ \\
\hline Whole parietal cortex & 1.01 & $1.04(0.97-1.11)$ \\
\hline Whole occipital cortex & $1 \cdot 18^{\star \star \star}$ & $1.00(0.87-1.12)$ \\
\hline Limbic cortex & 0.85 & $0.87(0.74-1.00)$ \\
\hline Caudate nucleus & 0.96 & $1.03(0.89-1 \cdot 16)$ \\
\hline Lentiform nucleus & 1.05 & $1.08(0.82-1.34)$ \\
\hline Basal ganglia $\int$ & 1.00 & $1.06(0.87-1.24)$ \\
\hline Thalamus & $1 \cdot 17$ & $1.09(0.86-1.31)$ \\
\hline Cerebellum & 0.94 & $0.94(0.70-1.16)$ \\
\hline
\end{tabular}

${ }^{\star} \mathrm{p}=0.05 ;{ }^{\star \star} \mathrm{p}<0.05 ;{ }^{\star \star \star} \mathrm{p}<0.01 v$ normal controls

+ Includes the frontobasal and the prefrontal lateral cortices.

Includes the prefrontal and the precentral cortices.

Includes the caudate and lentiform nuclei. 